CERN-TH/95-52

GeF-TH-3/1995

\title{
TOP QUARK DisTRIBUTIONS IN HADRONIC COLLISIONS
}

\author{
Stefano FRIXIONE[ \\ INFN, Sezione di Genova, Italy \\ Michelangelo L. MANGANO, 2] Paolo NASON, [ \\ Giovanni RIDOLFI t \\ CERN, TH Division, Geneva, Switzerland
}

\begin{abstract}
We present kinematical distributions for top quark pairs produced at the Tevatron $p \bar{p}$ Collider, as predicted within Next-to-Leading-Order QCD. We consider single and double-inclusive distributions, and compare our results to those obtained with the shower Monte Carlo HERWIG. We discuss the implications of our findings for experimental issues such as the measurement of the top quark mass.
\end{abstract}

CERN-TH/95-52

March 1995

\footnotetext{
${ }^{1}$ Address after June 1: ETH, Zürich, Switzerland

${ }^{2}$ On leave of absence from INFN, Pisa, Italy

${ }^{3}$ On leave of absence from INFN, Milano, Italy

${ }^{4}$ On leave of absence from INFN, Genova, Italy
} 
Now that the existence of the top quark has been firmly established via its detection in hadronic collisions [1, 2, 3], experimental studies will focus on the determination of its properties. In particular, the measurement of its mass and of the production cross section and distributions will certainly be among the first studies of interest. The top mass represents one of the crucial parameters for testing the Standard Model [4], while the production properties, should they display anomalies, could point to the existence of exotic phenomena [5].

Early estimates of the total production cross section for top quarks [6, 0] have later been updated [8]. In ref. [9] a partial resummation of leading logarithms from soft gluon emission has also been included.

In this Letter we present a set of kinematical distributions of relevance for the study of production dynamics, as obtained from full Next-to-Leading-Order (NLO) QCD perturbative calculations. We will consider inclusive $p_{T}$ and pseudorapidity $(\eta)$ distributions, as well as invariant mass $(M(t \bar{t}))$, transverse momentum $\left(P_{T}^{t \bar{t}}\right)$ and azimuthal correlations $(\Delta \phi)$ of the top quark pair.

The inclusive $p_{T}$ distribution is sensitive to channels such as $W g \rightarrow t \bar{b}$ [10], which are found to contribute with a small cross section, predominantly at low $p_{T}$. The invariant mass of the pair is an obvious probe of the possible existence of strongly coupled exotic resonances, such as technimesons [5]. The transverse momentum of the pair is an indication of the emission of hard hadronic jets in addition to those coming from the top decays. The presence of these additional jets generates potentially large combinatorial backgrounds to the reconstruction of the top mass peak from the decay products [2]. An accurate understanding of these backgrounds is in principle very important for a precise measurement of the top mass.

The NLO total, single and double inclusive cross sections for the production of heavy quarks in hadronic collisions have been calculated in references [6], [11] and [12], respectively. Since most experimental studies are performed using shower Monte Carlo event generators [13] to simulate the behaviour of the events in the detectors, we will compare our NLO results with those we obtained with HERWIG [14]. This is important in order to assess the reliability of the theoretical inputs used by the experiments. It has been recently pointed out 15 that there are possibly significant differences in the jet activity predicted by fixed-order parton level calculations and the results of HERWIG, with possible implications for the experimental mass measurement. We will discuss here a possible origin of these discrepancies.

For our calculations we use $m_{t}=176 \mathrm{GeV}$ (CDF, ref. [1]). Differences of \pm $15 \mathrm{GeV}$, approximately the current experimental error, do not affect the shapes of the distributions we present nor any of our conclusions. Our default set of parton densities is MRSA [16], and we explore a range of renormalization and factorization scales $\mu_{0} / 2<\mu_{\mathrm{R}}=\mu_{\mathrm{F}}<2 \mu_{0}$, with $\mu_{0}^{2}=m_{t}{ }^{2}+{p_{T}}^{2}\left(\mu_{0}{ }^{2}=m_{t}{ }^{2}+\left(\left(p_{T}\right)_{t}^{2}+\left(p_{T}\right)_{t}^{2}\right) / 2\right)$ for single (double) differential distributions.

Throughout our plots, we rescale the HERWIG calculations by the perturbative $K$ factor given by the ratio of the $\mathcal{O}\left(\alpha_{s}^{3}\right)$ and the $\mathcal{O}\left(\alpha_{s}^{2}\right)$ results. The $K$ factor is of the order of 1.3 for all choices of parameters. The distributions of $p_{T}, \eta$ and $M(t \bar{t})$ are shown in figures 1-3. The solid lines correspond to the NLO results obtained using $\mu_{\mathrm{R}}=\mu_{\mathrm{F}}=\mu_{0}$. The curves obtained using a different choice of scales, within the 


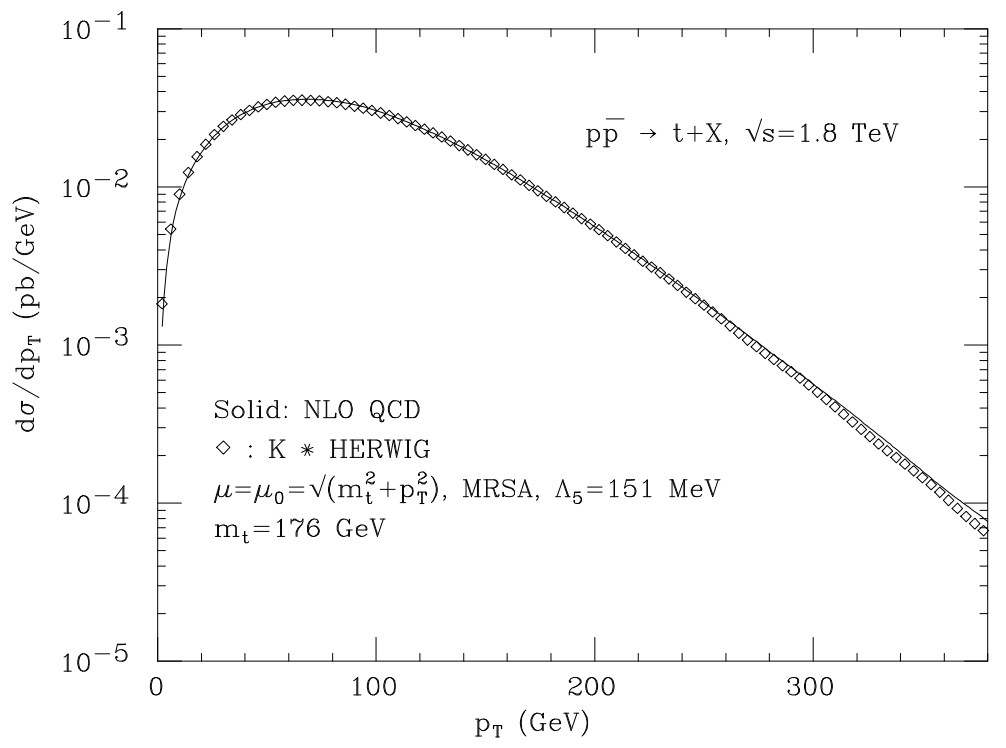

Figure 1: Inclusive $p_{T}$ distribution of the top quark.

range quoted earlier, result in an overall normalization change, by approximately $\pm 10 \%$. No change in the shapes is observed, and we therefore decided not to include these curves in the plots for the sake of clarity. The square points correspond to the HERWIG prediction for $\mu_{\mathrm{R}}=\mu_{0}$, rescaled by a $K$ factor equal to 1.34 .

The figures indicate clearly that distributions which are not trivial at leading order, such as $p_{T}, \eta$ and $M(t \bar{t})$, agree perfectly between the NLO and HERWIG calculations, at least for values of $p_{T}$ and $M(t \bar{t})$ not too large. This result follows naturally both from the observed stability of those distributions under radiative corrections regardless of the heavy quark mass [12], and from the large inertia of the top quark against the combination of higher order and non-perturbative corrections introduced by the Monte Carlo. At large $p_{T}$ and $M(t \bar{t})$, however, one starts observing a small deviation, due to the multiple gluon emission from the final state top quarks. This effect is not described by the NLO QCD calculation, which only includes one gluon emission. Multiple gluon emission becomes relevant for $p_{T} \gg m_{t}$, where it leads to a top quark fragmentation function softer than predicted by the fixed order NLO calculation. Due to the smallness of the cross section in these regions, large statistics will be needed in order to test this behaviour.

Those distributions which are trivial at leading order, $\Delta \phi$ and $P_{T}^{t \bar{t}}$, are viceversa most sensitive to multiple gluon emission from the initial state. This is because even small perturbations can smear a distribution which at leading order is represented by a delta function, which is the case of $P_{T}^{t \bar{t}}$ and $\Delta \phi$. The most visible effect is observed in $P_{T}^{t \bar{t}}$, Figure 4, where we include the NLO curves relative to the three choices of scales, $\mu_{\mathrm{R}}=\mu_{\mathrm{F}}=\mu_{0}$ (solid), $\mu_{0} / 2$ (dots) and $2 \mu_{0}$ (dashes). Contrary to the previous cases, significant differences in shape arise here among the three choices in the small $p_{T}$ region. The HERWIG result (normalized to the area of the solid curve) is also shown. The NLO and the HERWIG distributions assume the same shape only for $P_{T}^{t \bar{t}}$ larger than approximately $20 \mathrm{GeV}$. 


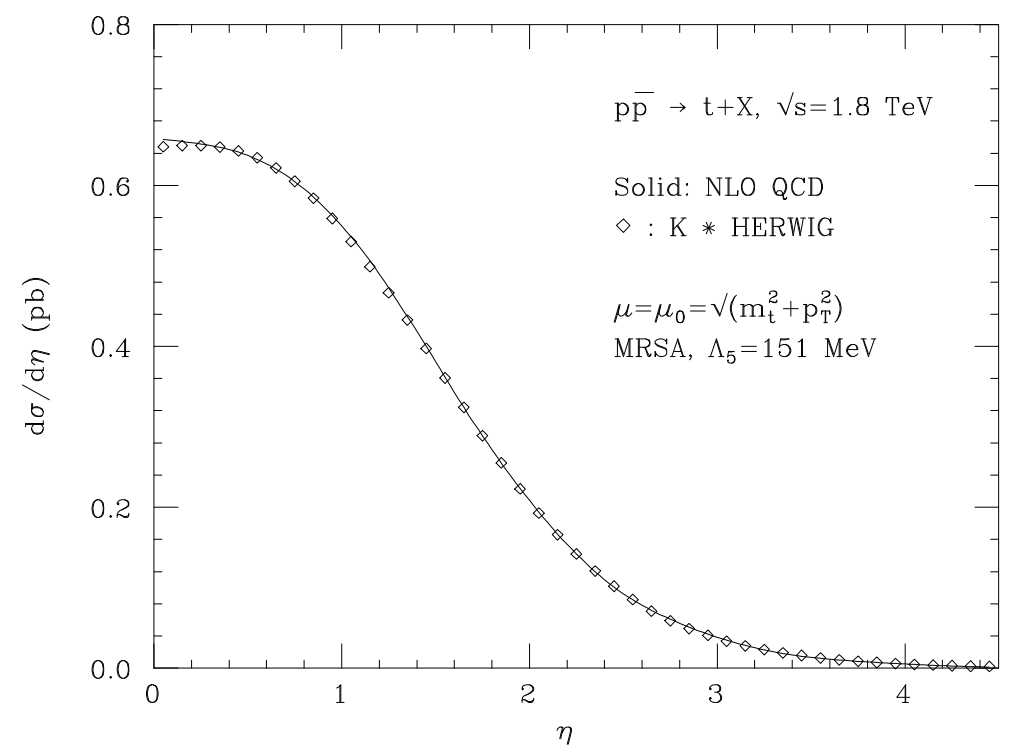

Figure 2: Inclusive pseudorapidity distribution of the top quark.

\begin{tabular}{lccc}
\hline \hline & $\left\langle P_{T}^{t \bar{t}}\right\rangle(\mathrm{GeV})$ & $\mathrm{F}\left(P_{T}^{t \bar{t}}>10 \mathrm{GeV}\right)(\%)$ & $\mathrm{F}\left(P_{T}^{t \bar{t}}>20 \mathrm{GeV}\right)(\%)$ \\
\hline \hline NLO QCD,$\mu_{\mathrm{R}}=\mu_{\mathrm{F}}=\mu_{0}$ & 11.9 & 30 & 15 \\
\hline NLO QCD,$\mu_{\mathrm{R}}=\mu_{\mathrm{F}}=2 \mu_{0}$ & 9.1 & 23 & 11 \\
\hline NLO QCD,$\mu_{\mathrm{R}}=\mu_{\mathrm{F}}=\mu_{0} / 2$ & 16.6 & 44 & 22 \\
\hline HERWIG, $\mu_{\mathrm{R}}=\mu_{\mathrm{F}}=\mu_{0}$ & 17.5 & 51 & 28 \\
\hline
\end{tabular}

Table 1: Average value of $P_{T}^{t \bar{t}}$ and fraction of the cross section with $P_{T}^{t \bar{t}}>10$ and $20 \mathrm{GeV}$ for different calculations.

To better quantify the differences, we quote in Table 1 the value of the average $P_{T}^{t \bar{t}}$ and of the fraction of the total cross section with $P_{T}^{t \bar{t}}>10,20 \mathrm{GeV}$ for the different curves appearing on the plot. First of all we notice the strong scale dependence of the results in Table 1. This is a first indication of the lack of reliability of the fixed order calculation for these particular quantities. Observe that the NLO result obtained with the lowest scale is closer to the HERWIG result. In fact, a lower scale is presumably more appropriate in this problem. This is because there are two relevant scales in this case: the mass of the top, and the transverse momentum cut. Since the production of top quarks takes place mainly through the exchange of a highly virtual gluon (with virtuality of the order of the top mass), while the emission of the jet takes place at the smaller scale of the jet transverse momentum, it is sensible to take in the $\mathcal{O}\left(\alpha_{s}^{3}\right)$ cross section two powers of $\alpha_{s}$ at the large scale $\mu_{0}$ and one at the transverse momentum of the jet. We have performed this exercise, and obtained $\sigma\left(P_{T}^{t \bar{t}}>10 \mathrm{GeV}\right)=1.987 \mathrm{pb}$, which is $43 \%$ of the total cross section 


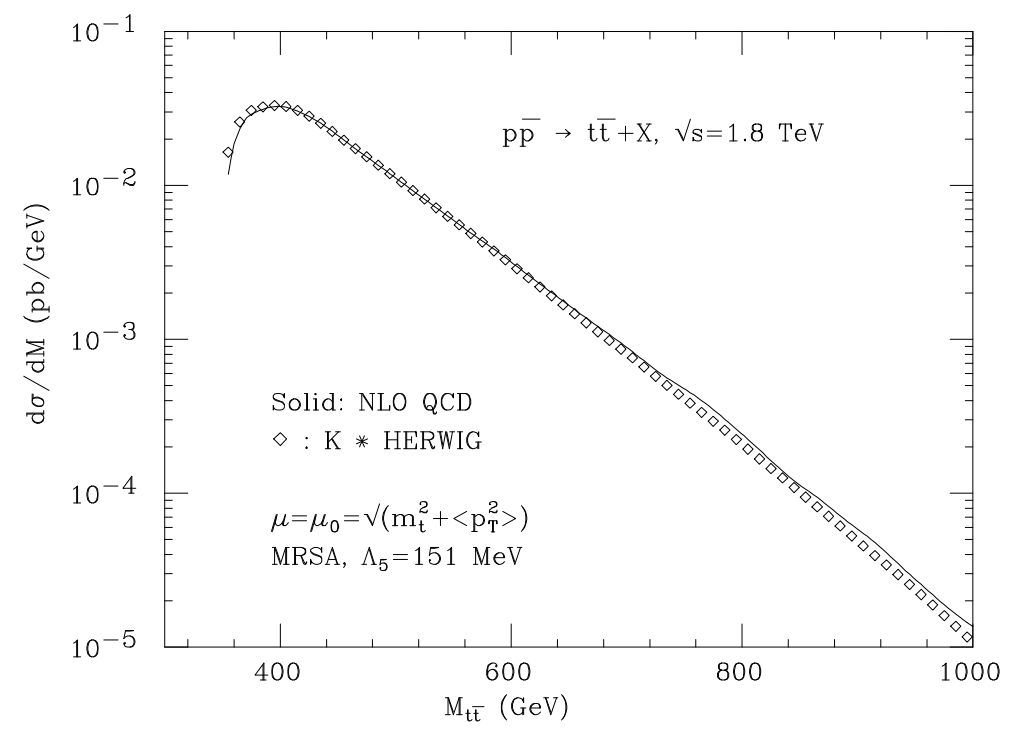

Figure 3: Invariant mass distribution of the $t \bar{t}$ pair.

computed at the scale $\mu_{0}$.

The high value of $\left\langle P_{T}^{t \bar{t}}\right\rangle$ for the NLO calculation indicates that the probability of emitting a gluon with transverse energies $k_{T}$ of the order of $10 \mathrm{GeV}$ is of order 1. Technically, this is related to the appearance of a large double logarithm $\mathcal{O}\left(\alpha_{s} \log ^{2}\left[m_{t}^{2} / k_{T}^{2}\right]\right)$. It is well known [17] that in these circumstances multiple gluon emission, corresponding to multiple powers of these double logs, needs to be resummed. A typical example can be found in $W$ and $Z$ production at small $k_{T}$ 18.

The Monte Carlo HERWIG performs the resummation of leading and some subleading double logarithms, faithfully reproducing the behaviour of the analytically resummed cross sections in known cases [14]. The large top mass extends the region where Sudakov effects are important up to $k_{T}$ values which are often considered within the domain of applicability of standard fixed-order perturbation theory.

We have implemented a leading logarithm version of the resummation procedure described by Collins Soper and Sterman in [18], and qualitatively reproduced the behaviour of the Monte Carlo" We therefore conclude that the parton level result (with the factorization and renormalization scale taken of the order of the mass of the top quark) is less reliable than the Monte Carlo in the small $P_{T}^{t \bar{t}}$ region, and gives a cross section (for $P_{T}^{t \bar{t}}>10 \mathrm{GeV}$ ) which is roughly $60 \%$ of what one would get with a more judicious choice of the scale, and with the inclusion of Sudakov effects. We also point out that no choice of scales in the NLO calculation would describe the detailed shape in the region $P_{T}^{t \bar{t}}<20 \mathrm{GeV}$. On the other hand, we expect the parton calculation to be more accurate in the large $P_{T}^{t \bar{t}}$ region, where the soft approximation used in the Monte Carlo breaks down.

A similar problem should be expected in the description of jet radiation during the production of any massive system, in particular $\mathrm{W}$ or $\mathrm{Z}$ boson pairs. This might affect parton level calculations of backgrounds to $t \bar{t}$ production in the dilepton plus

\footnotetext{
${ }^{5} \mathrm{~A}$ similar calculation in the context of $b$ pair production has been given in ref. 19].
} 


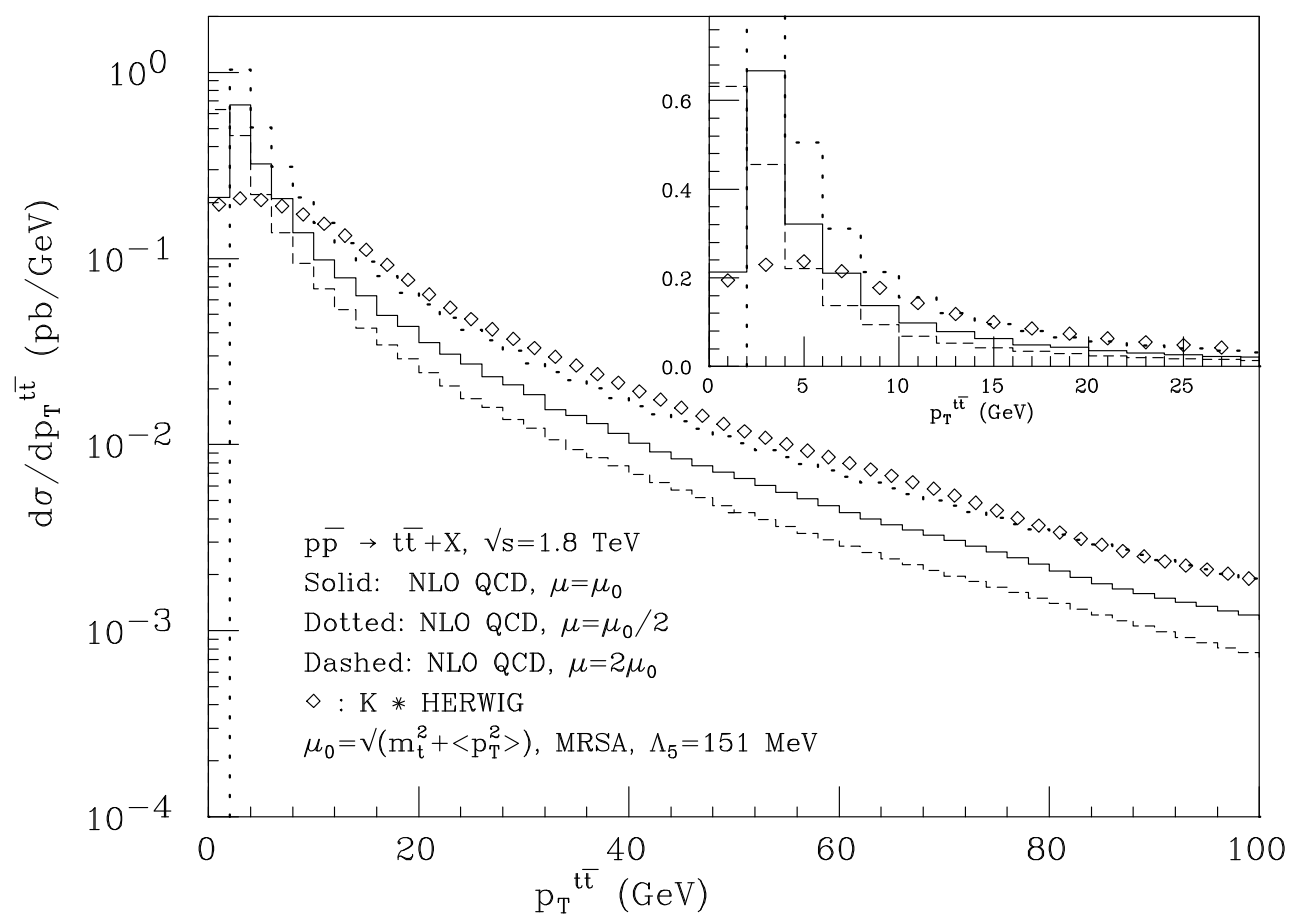

Figure 4: Transverse momentum distribution of the $t \bar{t}$ pair.

two jet channel. A simple estimate along the lines of what performed above for the top, sets the threshold below which fixed order perturbation theory becomes unreliable at about 10-15 GeV, which still corresponds to jets sufficiently energetic to be reconstructed as such by the Collider detectors. A confirmation of this estimate can be found in the study of resummation effects on the $p_{T}$ distribution of $\mathrm{Z}$ pairs presented in reference [20].

The observations made above partially explain the disagreement found in ref. 15] between the relative fraction of jets emitted during the production process and jets emitted from the $b$ quark resulting from the top decay. These authors found that their parton level calculation gave rise to a ratio smaller than HERWIG's. They interpreted it as a deficit in jet radiation off the final state $b$ quark within HERWIG. In order to make a more definite comparison with the results of ref. [15], we have computed the $t \bar{t}$ jet cross section with the same value of the parameters and the same set of cuts that they applied. We used therefore $m_{t}=174 \mathrm{GeV}, \mu_{0}=m_{t}$, $\left|\eta_{\text {jet }}\right|<2.5, P_{T}^{t \bar{t}}>10 \mathrm{GeV}$. In order to emulate the cuts they perform on the $b$ jets coming from top decays, we let the top quark decay isotropically, and imposed the additional cuts $\left|\eta_{\mathrm{b}}\right|<2.5$ and $p_{T}^{(b)}>10 \mathrm{GeV}$. We verified that these additional cuts affect the cross section by less than $3 \%$. We obtained a cross section of $1.30 \mathrm{pb}$, to be compared with the value of $1.0 \mathrm{pb}$ of ref. [15]. We have no explanation for this difference. From the considerations made previously, we expect that the inclusion of Sudakov effects should increase the cross section by roughly $66 \%$, leading to a value of $2.16 \mathrm{pb}$, more than a factor of two larger than the result of ref. [15].

Figure 5, finally, shows the $t \bar{t}$ azimuthal correlations. Notice that most of the shape discrepancies observed at $P_{T}^{t \bar{t}}<20 \mathrm{GeV}$ are forced here into the bin around 


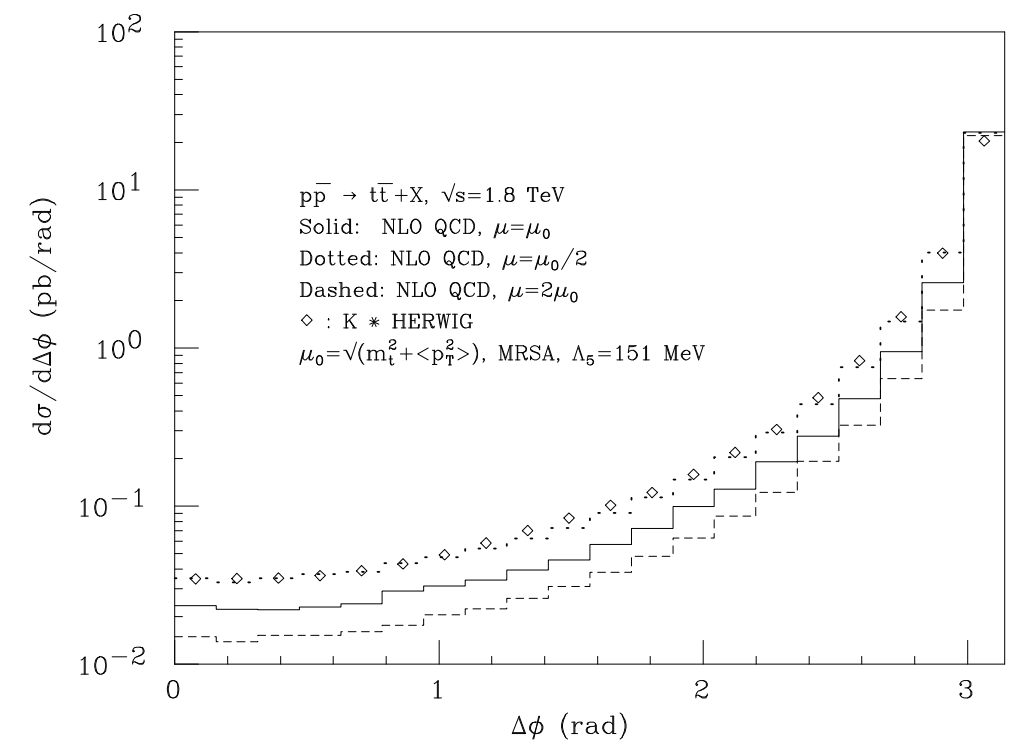

Figure 5: Azimuthal distance distribution of the $t \bar{t}$ pair.

the $\Delta \phi=\pi$ point. This is because even a $20 \mathrm{GeV}$ kick will only deflect the trajectory of the $t$ or $\bar{t}$ quark (each traveling with momentum of the order of $m_{t} / 2$ ), by about $1 / 10$ of a radian.

While completing this paper, a study of single inclusive distributions of top quarks at the Tevatron appeared [21]. These authors improved the NLO calculation with the inclusion of a partial resummation of leading soft logarithms [9]. Their results indicate that single inclusive distributions are only affected in overall normalization, but not in shape (at least for $p_{T}$ not much larger than $m_{t}$ ). This is consistent with the results presented here, and provides yet more confidence in the validity of the perturbative predictions applied to single inclusive quantities.

In concusion, we presented here a set of kinematic quantities which should be used to test the dynamics of top quark production in hadronic collisions. Most of these quantities were shown to be subject to very small theoretical uncertainty within NLO perturbative QCD, and to be rather insensitive to higher order or hadronization effects. One noticeable exception is the transverse momentum of the pair, which is in turn related to the spectrum of jets recoiling against the top pair. We found in our study that an accurate description of the region $P_{T}^{t \bar{t}}<20$ $\mathrm{GeV}$ requires the resummation of leading soft and collinear double logarithms, as implemented in appropriate shower Monte Carlo programs.

\section{References}

[1] F. Abe et al., CDF Collab., Fermilab-Pub-95-022-E., submitted to Phys. Rev. Lett., Febr 24 1995;

S. Abachi et al., D0 Collab., submitted to Phys. Rev. Lett., Febr 241995.

[2] F. Abe et al., CDF Collab., Phys. Rev. D50 (1994) 2966. 
[3] S. Abachi et al., D0 Collab., FERMILAB-PUB-94-354-E, to appear on Phys. Rev. Lett.

[4] The LEP Collaborations, ALEPH, DELPHI, L3 and OPAL, and the LEP Electroweak Working Group, CERN/PPE/94-187.

[5] E. Eichten and K. Lane, Phys. Lett. 327B (1994) 129;

C.T. Hill and S.J. Parke, Phys. Rev. D49 (1994) 4454.

[6] P. Nason, S. Dawson and R. K. Ellis, Nucl. Phys. B303 (1988) 607; W. Beenakker, H. Kuijf, W.L. van Neerven and J. Smith, Phys. Rev. D40 (1989) 54.

[7] G. Altarelli, M. Diemoz, G. Martinelli and P. Nason, Nucl. Phys. B308 (1988) 724.

[8] R.K. Ellis, Phys. Lett. 259B (1991) 492.

[9] E. Laenen, J. Smith and W.L. van Neerven, Nucl. Phys. B369 (1992) 543; Phys. Lett. 321B (1994) 254.

[10] S. Dawson, Nucl. Phys. B284 (1985) 449;

S. Willenbrock and D.A. Dicus, Phys. Rev. D34 (1986) 155;

C.P. Yuan, Phys. Rev. D41 (1990) 42;

R.K. Ellis and S.J. Parke, Phys. Rev. D46 (1992) 3785;

G. Bordes and B. van Eijk, Nucl. Phys. B435 (1995) 23.

[11] P. Nason, S. Dawson and R. K. Ellis, Nucl. Phys. B327 (1988) 49; W. Beenakker et al., Nucl. Phys. B351 (1991) 507.

[12] M. Mangano, P. Nason and G. Ridolfi, Nucl. Phys. B373 (1992) 295.

[13] B.R. Webber, Annu. Rev. Nucl. Part. Sci. 36(1986)253;

R. Odorico, Computer Phys. Comm. 32(1984)139;

G. Marchesini and B.R. Webber, Nucl. Phys. B238 (1984) 1;

B.R. Webber, Nucl. Phys. B238 (1984) 492;

H.-U. Bengtsson and G. Ingelman, Computer Phys. Comm. 34(1985)251;

R.D. Field, Nucl. Phys. B264 (1986) 687;

F. Paige and S.D. Protopopescu, Brookhaven report BNL-38034 (1986);

T. Sjöstrand and M. Bengtsson, Computer Phys. Comm. 43(1987)367;

B. Andersson, G. Gustafson and B. Nilsson-Almqvist, Nucl. Phys. B281 (1987) 289.

[14] G. Marchesini and B.R. Webber, Nucl. Phys. B310 (1988) 461. In this work we used version 5.8 of the program, after modifying the default choice of scale for the hard process to $\mu_{\mathrm{R}}=\mu_{\mathrm{F}}=\mu_{0}$.

[15] L.H. Orr, T. Stelzer and W.J. Stirling, DTP/94/112, hep-ph/9412294.

[16] A.D. Martin, R.G. Roberts and W.J. Stirling, Phys. Rev. D50 (1994) 6734. 
[17] Yu.L. Dokshitzer, D.I. Dyakonov and S.I. Troyan, Phys. Rep. 58 (1980) 271; G. Parisi and R. Petronzio, Nucl. Phys. B154 (1979) 427.

[18] G. Altarelli, R.K. Ellis, M. Greco and G. Martinelli, Nucl. Phys. B246 (1984) 12 ;

J.C. Collins, D.E. Soper and G. Sterman, Nucl. Phys. B250 (1985) 199.

[19] E. Berger and R. Meng, Phys. Rev. D49 (1994) 3248.

[20] T. Han, R. Meng and J. Ohnemus, Nucl. Phys. B384 (1992) 59.

[21] N. Kidonakis and J. Smith, ITP-SB-94-63, hep-ph/9502341. 